\title{
Machine Learning based Noise Estimation in Optical Fiber Communication Networks
}

\author{
(Invited Paper) \\ Seb J. Savory and F.J. Vaquero Caballero \\ Electrical Engineering Division, Department of Engineering, University of Cambridge, Cambridge CB3 OFA, U.K. \\ Email: sjs1001@cam.ac.uk and fjv24@cam.ac.uk
}

\begin{abstract}
In this paper, we discuss a machine learning based approach to jointly estimating both linear and nonlinear noise contributions in an optical fiber communication link. We will expound the rational for utilizing machine learning for this problem, before discussing current progress and then concluding with future research directions.
\end{abstract}

\section{INTRODUCTION}

The capacity of an optical fiber communication system is fundamentally limited by noise and nonlinearity. For these systems there is an optimal intensity of light to launch into the optical fiber with operatation at this optimal requiring both the noise and nonlinearity to be estimated. In a typical optical fiber communication system the amplified sponteneous emission (ASE) from the in line erbrium doped fiber amplifiers (EDFA) dominates and while Possionian in nature can be modeled as an additive white Gaussian noise (AWGN)[1]. In order to increase the signal to noise ratio (SNR), the launch power into the fiber is increased until nonlinear phenomena are observed. Since the nonlinear effects degrade the SNR an optimum launch power exists at which the maximum available SNR is observed.

The dominant nonlinear phenomena for optical fiber communication systems is the Kerr effect which causes the refractive index to change with intensity. Even though the change in the refractive index is small (less than one part in a billion) for a $100 \mathrm{GbE}$ channel the Kerr effect limits the performace at milliwatt power levels being commensurate with the power available from a semiconductor laser diode. Owing to the chromatic dispersion present in the fiber, the signal can be spread over hundreds of symbol periods resulting in the signal having a Gaussian like property. This allows the fiber nonlinearity to be modeled as a second AWGN with a variance that varies as the cube of the input power[2]. While this simplification allows for approximate calculations of performance, in practice the noise is neither Gaussian nor white, depending on the modulation format, the spectral shape of the waveform and the interaction between the nonlinearity and the chromatic dispersion in the fiber. Nonetheless for the optical fiber communication channel in principle it is possible to computationally solve the vector nonlinear partial differential equations to calculate the expected performance and the associated optimal launch power.

In simulation the situation is idealized, with all of the fiber parameters known with the optical amplifiers giving uniform gain and hence noise for all wavelength channels. In practice however amplifiers have gain ripple and gain tilt such that different channels experience different gain and noise such that there is uncertainty in the linear AWGN. Likewise even if the fiber parameters were known exactly (which in general they are not), there is uncertaintly in the power levels, resulting in uncertainty in the nonlinear noise contribution to the performance. Furthermore in an optical network wavelength channels are routed in the optical domain such that the nonlinear interaction with other channels varies spatially throughout the network as different channels are added or dropped along a path within the network. The final complication is that the transceiver itself adds noise, ranging from quantization noise in the data converters to noise from tracking errors in the various digital signal processing algorithms in the receiver. From this we observe that the estimation of noise in a practical optical fiber communication network is hindered by uncertainty relating to the channel and as such a framework for estimation in the presence of uncertainty is required.

\section{Why USE MACHINE LEARNING?}

As outlined in the introduction, the estimation of noise for the purpose of determining the signal to noise ratio is hindered by the presence of uncertainty. We propose to use machine learning as a means of estimation in the presence of uncertainty, initially focusing on neural networks as a means of obtaining the nonlinear relationship between the SNR and the various input parameters (such as the auto-correlation function, total accumulated chromatic dispersion etc.).

Optical performance monitoring is a well established research area that attempts to monitor optical transmission systems with a view to assessing the quality of a transmission link[3], albeit herein we focus on the estimation of linear and nonlinear noise contributions. Classical approaches include fitting a three parameter model[4] to separate the nonlinear and linear noise contributions or estimating the ASE noise component from an optical spectrum analyzer[5].

Recently machine learning techniques have emerged for robust estimation of the linear and nonlinear contributions to SNR based on neural network based regression[6], [7], [8], [9]. Herein we focus on neural networks, providing a basis for robust joint estimation of the linear and nonlinear noise contributions building on our previous work[9]. 


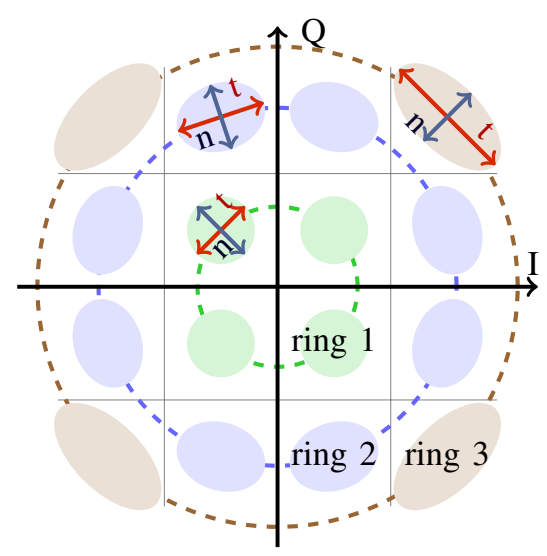

Fig. 1: 16-QAM signal including $\mathbf{t}$ and $\mathbf{n}$ components.

\section{EXAMPLE APPLICATION: DP-16QAM}

By way of an introduction to joint estimation of linear and nonlinear estimation we consider a dual polarization (DP) 16-ary quadrature amplitude modulation (QAM) format. As illustrated in Fig. 1 in one polarization the 16 uniformly distributed points become slightly elliptical due to nonlinear phase noise[10]. We exploit this feature in order to estimate and distinguish the linear and nonlinear noise contributions.

In Fig 1, we show a 16-QAM constellation with the subdivision of its noise variance into its normal, $\mathbf{n}$, and tangential, t, components, noting that the nonlinear phase noise affects only the tangential component. The SNR can be expressed as:

$$
S N R=\frac{1}{\langle\mathbf{n}\rangle+\langle\mathbf{t}\rangle}
$$

where $\langle\mathbf{n}\rangle=\left(N_{1}+2 N_{2}+N_{3}\right) / 4$ and $\langle\mathbf{t}\rangle=\left(T_{1}+2 T_{2}+T_{3}\right) / 4$ where $N_{i}$ and $T_{i}$ are the averages for each power ring $(i=\{1,2,3\})$. Additionally, we exploit the correlation of nonlinearities through the use of the amplitude noise covariance (ANC) and its accumulated logarithm version (ALANC):

$$
\begin{aligned}
& A N C_{i j}(m)=\operatorname{cov}\left(\Delta s_{k, i}, \Delta s_{k+m, j}\right), \\
& A L A N C_{i j}=10 \log _{10}\left(1 / \sum_{m=1}^{6}\left|A N C_{i j}(m)\right|\right), i, j \in\{x, y\}
\end{aligned}
$$

where $\Delta s_{k, i}$ is defined as the difference between received and expected symbols $\left|s_{k, i}\right|-\left|\hat{s}_{k, i}\right|$ in the $i$-polarization.

A small neural network (one hidden layer consisting of 7 neurons) is trained to find the relationship between the parameters. A hyperbolic tangent sigmoid is used as the nonlinear transfer function for the hidden neurons. The input to the neural networks are $\left\{N_{1}, N_{2}, N_{3}, T_{1}, T_{2}, T_{3}\right\}$, the accumulated chromatic dispersion, $A L A N C_{x x}, A L A N C_{x y}$, and the number of WDM channels. The outputs of the neural network are $S N R_{L I N}$ and $S N R_{N L I}$.

The transmission realizations consist of 2160 datasets, involving different types of fibers, number of spans, number of WDM channels, and different amounts of linear noise loaded in the receiver[9]. Neural network based regression gave a standard deviation of $0.23 \mathrm{~dB}$ for both $S N R_{L I N}$ and $S N R_{N L I}$ demonstrating the efficacy of the technique.
As the transmission distance increases, the eccentricity of the nonlinearities decreases, consequently the difference between the normal and tangential components becomes marginal and the noise estimation inference will suffer greater uncertainty for longer distances. Lower order modulation formats present similar limitations since the nonlinear phase noise is not a dominant nonlinear contribution[10].

\section{CONClusion AND Future DiRECTIONS}

In this paper we introduced the use of machine learning for the joint estimation of linear and nonlinear noise and discussed its limitations. While neural networks provide a basis for initial investigations, other machine learning techniques may provide more insight when dealing with uncertainties. This is of particular interest as we move towards metrics capable of distinguishing between various linear and nonlinear noise components for any modulation format and reach. Ultimately classical approaches based on regression, machine learning and deep learning will need to be compared in terms of their complexity, accuracy and training data required but also the information conveyed to a network operating system as we move towards a cognitive optical network.

\section{ACKNOWLEDGMENTS}

The authors gratefully acknowledge the donation of equipment, funding and support from Ciena. This research was performed under the auspices of a Ciena university collaborative research grant. S. J. Savory also acknowledges support from UK EPSRC (through the project INSIGHT EP/L026155/2).

\section{REFERENCES}

[1] J. P. Gordon, "Quantum Effects in Communications Systems," Proceedings of the IRE, vol. 50, pp. 1898-1908, Sept 1962.

[2] P. Poggiolini, "The GN Model of Non-Linear Propagation in Uncompensated Coherent Optical Systems," Journal of Lightwave Technology, vol. 30, pp. 3857-3879, Dec 2012.

[3] X. Zhou and C. Xie, Enabling Technologies for High Spectral-efficiency Coherent Optical Communication Networks. Wiley, 2016.

[4] H. M. Chin, D. Charlton, A. Borowiec, M. Reimer, C. Laperle, M. OSullivan, and S. J. Savory, "Probabilistic Design of Optical Transmission Systems," Journal of Lightwave Technology, vol. 35, pp. 931-940, Feb 2017.

[5] D. Gariepy, S. Searcy, G. He, and S. Tibuleac, "Novel OSNR Measurement Techniques for Coherent-Detection Systems," in Optical Fiber Communication Conference, 2018.

[6] A. S. Kashi, Q. Zhuge, J. C. Cartledge, A. Borowiec, D. Charlton, C. Laperle, and M. O'Sullivan, "Fiber Nonlinear Noise-to-Signal Ratio Monitoring Using Artificial Neural Networks," European Conference on Optical Communication, 2017.

[7] F. N. Khan, K. Zhong, X. Zhou, W. H. Al-Arashi, C. Yu, C. Lu, and A. P. T. Lau, "Joint OSNR monitoring and modulation format identification in digital coherent receivers using deep neural networks," Optics Express, vol. 25, no. 15, pp. 17767-17776, 2017.

[8] S. Yan, F. N. Khan, A. Mavromatis, D. Gkounis, and Q. Fan, "Field trial of Machine-Learning-assisted and SDN-based Optical Network Planning with Network-Scale Monitoring Database," in European Conference on Optical Communication, 2017.

[9] F. J. Vaquero Caballero, D. J. Ives, Q. Zhuge, M. O. Sullivan, and S. J. Savory, "Joint Estimation of Linear and Non-linear Signal-to-Noise Ratio based on Neural Networks," in Optical Fiber Communication Conference, 2018.

[10] R. Dar, M. Feder, A. Mecozzi, and M. Shtaif, "Pulse Collision Picture of Inter-Channel Nonlinear Interference in Fiber-Optic Communications," Journal of Lightwave Technology, vol. 34, no. 2, pp. 593-607, 2016. 